\title{
RF-Care: Device-Free Posture Recognition for Elderly People Using A Passive RFID Tag Array
}

\author{
Lina Yao ${ }^{1}$, Quan Z. Sheng ${ }^{1}$, Wenjie Ruan ${ }^{1}$, Tao $\mathrm{Gu}^{2}$, \\ Xue Li $^{3}$, Nickolas J.G. Falkner ${ }^{1}$, and Zhi Yang ${ }^{1}$ \\ ${ }^{1}$ School of Computer Science, The University of Adelaide, Adelaide, SA 5005, Australia \\ \{lina.yao, michael.sheng, wenjie.ruan, nick.falkner, zhi.yang\}@adelaide.edu.au \\ ${ }^{2}$ School of CS \& IT, RMIT University, Melbourne, VIC 3000, Australia \\ tao.gu@rmit.edu.au \\ ${ }^{3}$ School of ITEE, The University of Queensland, Brisbane, QLD 4072, Australia \\ xueli@itee.uq.edu.au
}

\begin{abstract}
Activity recognition is a fundamental research topic for a wide range of important applications such as fall detection for elderly people. Existing techniques mainly rely on wearable sensors, which may not be reliable and practical in real-world situations since people often forget to wear these sensors. For this reason, devicefree activity recognition has gained the popularity in recent years. In this paper, we propose an RFID (radio frequency identification) based, device-free posture recognition system. More specifically, we analyze Received Signal Strength Indicator (RSSI) signal patterns from an RFID tag array, and systematically examine the impact of tag configuration on system performance. On top of selected optimal subset of tags, we study the challenges on posture recognition. Apart from exploring posture classification, we specially propose to infer posture transitions via Dirichlet Process Gaussian Mixture Model (DPGMM) based Hidden Markov Model (HMM), which effectively captures the nature of uncertainty caused by signal strength varieties during posture transitions. We run a pilot study to evaluate our system with 12 orientation-sensitive postures and a series of posture change sequences. We conduct extensive experiments in both lab and real-life home environments. The results demonstrate that our system achieves high accuracy in both environments, which holds the potential to support assisted living of elderly people.
\end{abstract}

\section{Categories and Subject Descriptors}

C.4 [Special-Purpose and Application-Based System ]: Realtime and RFID-based system

\section{Keywords}

Activity Recognition, Device-Free, Passive RFID, Posture Detection, Posture Transition

\section{INTRODUCTION}

Activity recognition is one of the most promising research topics in pervasive computing applications. With recent advances in embedded sensors and wireless technologies, it has become possible to develop a wide range of applications such as surveillance [9], ambient assisted living [20,6], and remote health monitoring and intervention. For instance, by monitoring the activities of an older adult with dementia, we can track how completely and consistently her/his daily routines are performed and determine when assistance is needed.

Over the years, significant research work has been devoted to recognize human activities. Computer vision based approaches are one of such key solutions. However, such solutions usually demand high computational cost, and the performance depends much on lighting conditions (e.g., does not work well at night). In addition, user privacy is a big concern as camera is often considered intrusive. With the growing maturity of sensor, radio-frequency identification (RFID), and wireless networking technologies, activity recognition based on inertial, unobstrusive sensor readings has become popular [13, 11, 27, 26, 19, 5]. Sensor-based approaches require user to wear sensor devices which also need regular maintenance such as replacing batteries. In addition, these approaches may not be reliable, especially for older people with cognitive disability since they tend to forget wearing them. Recent years, we have seen a growing interest in device-free activity recognition, in which sensors are deployed to environments rather than worn by users. Radio signal fluctuations induced by user's movements can be analyzed to recognize activities [28, 21, 30, 23, 29]. Existing device-free systems depend on dense deployment of Zigbee devices in the environment, which can be costly, and still require battery maintenance. For example, in [10], the authors set up a system where a Zigbee transmitter is placed in the center of a room, and receivers (i.e., sensor array) are placed around the room and close to wall.

In this paper, we propose a novel device-free posture recognition system based on RFID. We use passive RFID tags which have some advantages than battery-powered sensors due to low cost and maintenance free. Figure 1 illustrates our system setup. First, we are interested in investigating RFID tag configuration issues from the system design point of view, particularly the issues on RFID tag placement (i.e., where and how to place tags) and tag selection (i.e., finding an optimal set of tags). Due to the complex nature of radio signals in an indoor environment, these issues can be crucial to system performance. Existing research findings show that pas- 


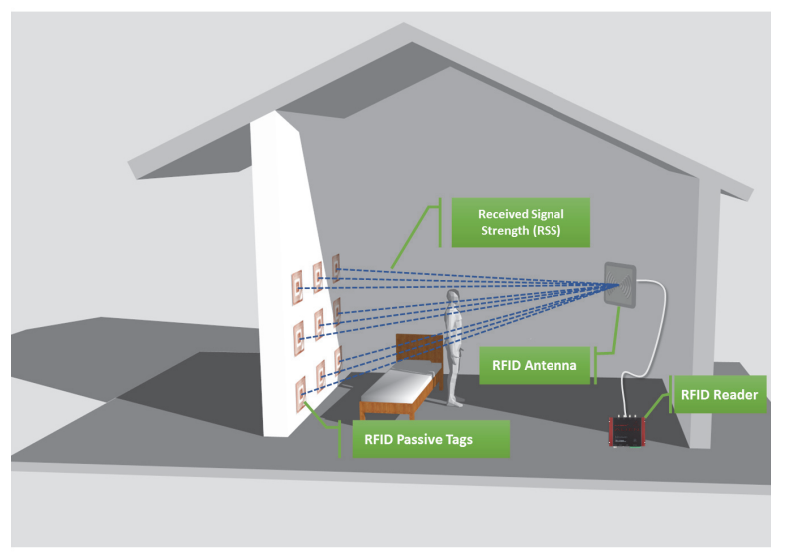

Figure 1: System overview: a user performs different postures in a room setting equipped with an RFID array.

sive RFID tags can cause some unpredictable effects if they are not placed properly, e.g., significant signal loss or fading if two tags are put in a certain distance [8]. Similar studies have been done to explore the optimal tag configuration in indoor localization. For example, a recent study by Wagner et al. [25] shows an optimal tag placement to alleviate inaccuracy caused by the variability of RSSI.

In our work, we focus on the study in the context of posture recognition which requires capturing fine-grained body movement compared to indoor localization. We first study the best possible tag placement, and examine and eliminate the redundant correlations of tags to find an optimal set of tags. Second, to evaluate the performance of our pure passive RFID system handling highly dynamic variations of RSSI during posture transitions, we propose a Dirichlet Process Gaussian Mixture Model (DPGMM) with Hidden Markov Model (HMM) model to detect a sequence of different postures (e.g., from sitting to standing to falling), respectively. The main contributions of this paper are summarized as follows.

- We propose a novel device-free posture recognition system based on passive RFID tags, which is low-cost, unobtrusive, and maintenance free. The proposed system has many potential applications, especially for elderly people or people with cognitive impairment.

- We investigate both tag placement and selection issues, aiming to discover the basic design principle for deploying our system in indoor settings. The study determines an optimal setting of tag array to achieve the best system performance with less computational demand. We propose and compare various tag selection techniques (e.g., F statistics, relief $\mathrm{F}$, random forest, etc) to identify and eliminate redundant tags.

- We deploy our system in an indoor setting, and conduct experiments to validate our system. The experimental results with a single subject in both a lab and a home settings show that the system achieves an accuracy of $98 \%$ on average for 9 tags and one reader in dealing with steady posture recognition, and over $70 \%$ accuracy in dealing with a sequence of posture transitions, which result in more disturbance and noisy data of RSSI. Our experimental results demonstrate that the proposed system has good potential to be developed further and deployed in practice.

The rest of the paper is organized as follows. In Section 2, we discuss some potential applications, and present our key observa-

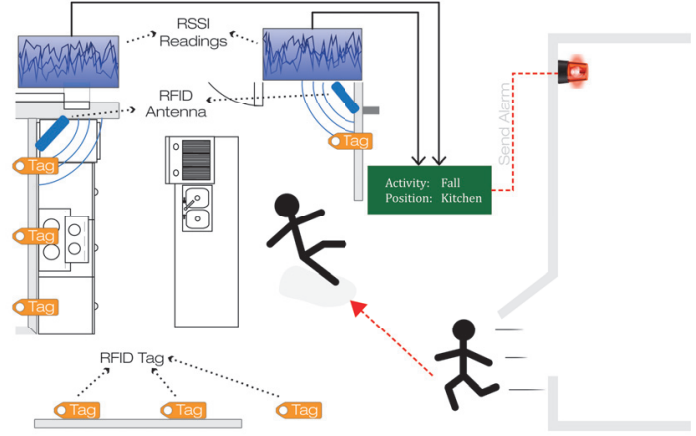

Figure 2: An illustraton of fall of an older resident

tions to motivate our work. We describe the system design in Section 3. In Section 4, we report our experimental results. We review the related work in Section 5 and wrap up the paper in Section 6.

\section{BACKGROUND}

In this section, we first discuss several representative applications that can benefit from our system. We then formulate the research problem.

\subsection{Application Scenario}

The system we present in this paper can be applied to posture monitoring and activity recognition in general, particularly for elderly people or people with cognitive impairment. Figure 2 illustrates a home scenario where an older adult accidentally falls down, and the system detects this abnormal action and sends an alarm immediately to caregiver for assistance.

\subsection{Observations and Problem Formulation}

Figure 1 depicts a typical system setup in a room setting, where an RFID tag array is deployed on the wall, and RFID antenna are placed on the other side, facing these tags. When a person performs different activities in the room, the system collects RSSI values and discovers varied fluctuation patterns. The motivation of our work arises from the following observations.

It is known that RSSI signal exhibits a variety of complicated phenomena in indoor environments due to signal reflection, diffraction, and scattering. This is often affected by the propagation environment, the tagged object properties, and human movements in the signal coverage area. It is difficult to model RSSI signal approximately by a location-dependent path loss model. Figure 3 shows RSSI signal values of passive RFID tags, which fluctuate with different postures. Although RSSI shows uncertainty and non-linear distributed patterns, we discover that the variations of RSSI values allow us to distinguish different postures. Figure 4 shows the distinctive changes of RSSI values of the tag array (e.g., 9 passive RFID tags) according to different postures that a subject performs.

From these observations, we believe that RSSI signals of passive RFID tags embody different patterns for different postures, which can be further exploited for our posture recognition task. We therefore formulate our problem in this work as follows.

Let $\mathcal{O} \subset \mathbb{R}^{d}$ ( $d$ is the number of tags) be the domain of observable RSSI $o$ and $\mathcal{L} \in\{1, \ldots, K\} \subset \mathbb{R}$ be the domain of output posture label $l$. Suppose we have $n$ RSSI and posture label pairs $\left\{\left(\mathbf{o}_{i}, l_{i}\right) \mid \mathbf{o}_{i} \in \mathcal{O}, l_{i} \in \mathcal{L}, i=1, \ldots, n\right\}$. The training dataset can be represented as:

$$
\begin{array}{r}
\mathbf{O}=\left[\mathbf{o}_{1}, \ldots, \mathbf{o}_{n}\right] \in \mathbb{R}^{d \times n} \\
\mathbf{l}=\left[l_{1}, \ldots, l_{n}\right]^{T} \in \mathbb{R}^{n}
\end{array}
$$




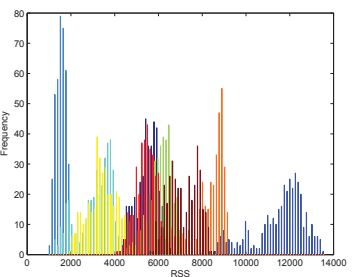

(a)

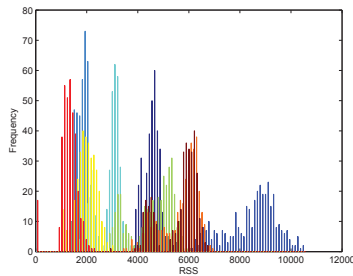

(b)
Figure 3: (a) Histogram of RSSI from posture sitting leaning left; (b) Histogram of RSSI from posture sitting leaning right

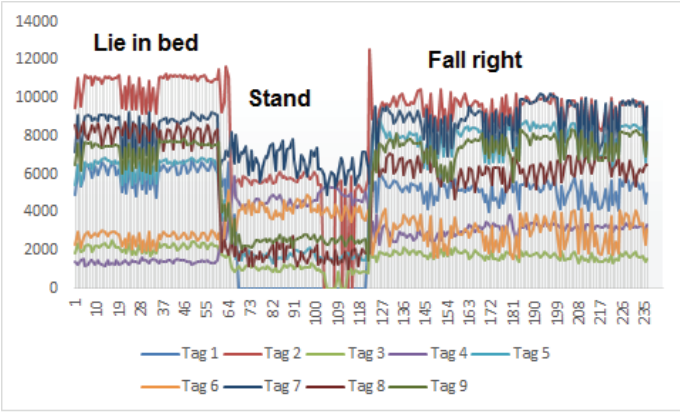

Figure 4: Different RSSI patterns of different postures

In this paper, we are interested to investigate the following two questions.

Problem 1 (Tag Placement and Selection). Given an RFID tag array, how to place them in a room setting and find the optimal subset of tags to achieve the best performance.

Problem 2 (Posture Recognition). Given the RSSI values we observe, how to recognize a subject's postures.

\section{THE PROPOSED APPROACH}

In this section, we first present the tag placement and selection, followed by the technical details on the posture recognition.

\subsection{Tag Placement and Selection}

Tag Placement. To realize posture recognition, the first essential challenge for us is how to set up tags in an indoor setting to obtain the best performance. We first describe some intuitions of tag placement in this work. There are two main reasons why we place tags as an array:

- We conduct empirical studies on different forms of placing tags, such as arranging tags as a single line on the wall. According to our results, single-line tag placement is capable of capturing signal variations, but it may fail to detect finegrained body movements, such as sitting leaning right or left. Furthermore, it is also not sensitive to capture the signal variations caused by subjects with different heights.

- To achieve better accuracy and higher sensitivity, we increase single-line tag placement to multiple lines, eventually forming an array. Different lines correspond to different parts of human body. For instance, the upper line of tags would be expected to reflect the variations from upper human body like waving arms or shaking head, and the middle line of tags would be more sensitive to movements of torso, and the bottom line of tags are supposed to have more response to lower

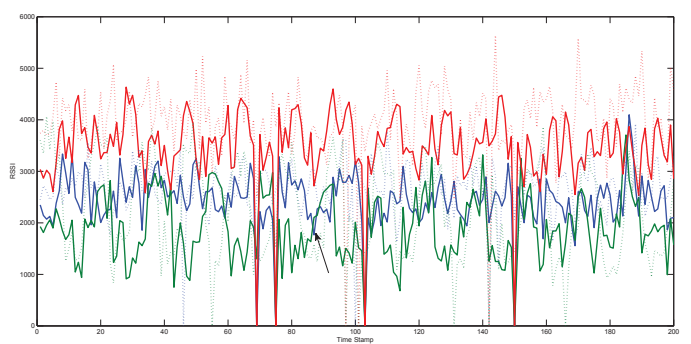

(a)

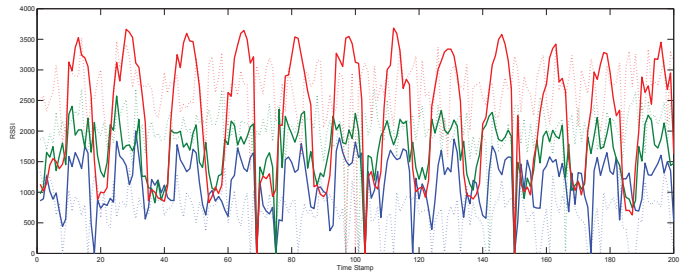

(b)

Figure 5: Illustration of RSSI fluctuations of falling right and falling left: RSSIs of tag 1 , tag 2 and tag 3 (top) and RSSIs of $\operatorname{tag} 7, \operatorname{tag} 8$ and tag 9 .

body movements such as falling etc. In this way, we may perform more robust posture recognition with the collected full spectrum of RSSI variations.

As shown in Figure 5, the top one shows the RSSI fluctuations of three tags (tag 1, tag 2 and tag 3 are placed as a single line shown in Figure 13) and the lines indicate RSSI variations of falling right, and dash lines indicate RSSI variations of falling left. The bottom one shows the RSSI fluctuations of three tags (tag 7, tag 8 and tag 9 are arranged as a single line) and the solid lines indicate RSSI variations of sitting right, and dash lines indicate RSSI variations of sitting left. We can observe the fluctuations of tag 1, tag 2 and tag 3 are not quite helpful for reflecting different orientation falls as they do not show significant difference, on the other hand, tag 7 , tag 8 and tag 9 can distinguish falling right and falling left better. The reason lies in fall action happens on the lower body, the lower location of tag 7 , tag 8 and tag 9 can be more sensitive to such actions compared with tag 1, tag 2 and tag 3 in upper location. To capture the RSSI variations in all aspects, we use multiple lined up tags forming a tag array in this work. Existing works such as [10] also show that placing sensors as an array can realize activity recognition with good accuracy.

Tag Selection. With the placement of tag array on the wall, we now focus on studying the correlations between tags while detecting postures. As shown in Figure 6 (a), the RSSI values of tag 1 and tag 2 are highly correlated with each other, implying that one of them is redundant. Figure 6 (b) illustrates that RSSI values of tag 1 and tag 9 successfully divide the RSSI data space from the series of postures like standing straight, sitting straight, and lying on bed. We also examine the redundant correlations among three tags. For example, Figure 6 (d) shows that the RSSI values of tag 3 , tag 5 and tag 9 can distinguish the listed postures, while the RSSI values of tag 3 , tag 4 and tag 6 are highly correlated, as shown in Figure 6 (c).

From above observations, to eliminate the redundancy and identify discriminative usefulness of tags and choose the most appropriate selection method to validate our proposed system, we examine a series of techniques to select a salient subset of tags to determine 


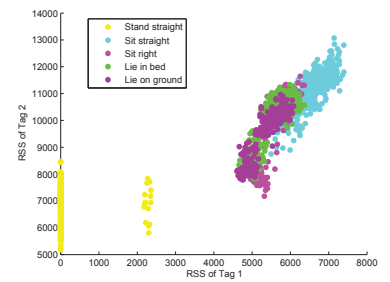

(a)

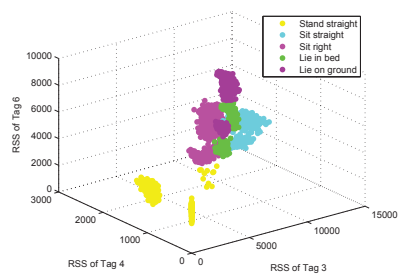

(c)

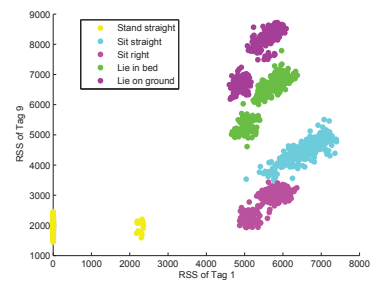

(b)

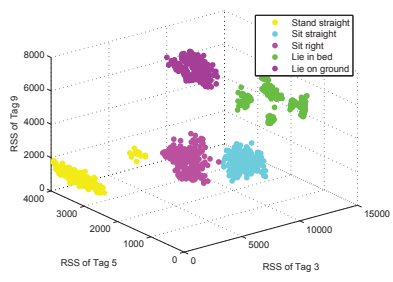

(d)
Figure 6: Illustrative examples of tag correlations

the optimal tag array configuration in our system.

$F$-Statistics. It is to measure the discrimination of multiple sets of real numbers and is calculated using:

$$
F_{i}=\frac{\sum_{j=1}^{l}\left(\overline{\mathbf{o}}_{i}^{j}-\overline{\mathbf{o}}_{i}\right)^{2}}{\sum_{j=1}^{l} \frac{1}{n_{j}-1} \sum_{k=1}^{n_{j}}\left(\mathbf{o}_{k, i}^{j}-\overline{\mathbf{o}}_{i}^{j}\right)^{2}}
$$

where $l$ is the number of posture classes, $n_{j}$ is the number of samples in $j^{\text {th }}$ posture class. $\overline{\mathbf{o}}_{i}$ denotes the mean value of tag $i$ in the training dataset. $\overline{\mathbf{o}}_{i}^{j}$ is the mean value of $i^{t h}$ tag in the $j^{t h}$ posture class. The numerator indicates the discrimination between positive and negative sets, and the denominator indicates the one within each of the two sets. The larger the F-score is, the more likely this tag is discriminative in posture recognition.

Relief $F$. This technique estimates the relevance of features according to how well their values distinguish between the data points of the same and different posture classes that are close each other. It computes a weight for each tag to quantify its merit. This weight is updated for the RSSI samples presented in each posture class, according to the evaluation function:

$$
\begin{aligned}
w_{i} & =w_{i}+\sum_{j \in \mathcal{L}, j \neq l\left(\mathbf{o}_{i}\right)} \frac{P\left(l_{j}\right)}{1-P\left(l_{j}\right)} \mid \mathbf{o}_{i}-\text { nearmiss }_{i}^{j}\left(\mathbf{o}_{i}\right) \mid \\
& -\mid \mathbf{o}_{i}-\text { nearhit }_{i}\left(\mathbf{o}_{i}\right) \mid
\end{aligned}
$$

where $l$ is the number of posture classes. nearmiss $j\left(\mathbf{o}_{i}\right)$ and nearhit $_{i}\left(\mathbf{o}_{i}\right)$ denote the nearest RSSI samples to $\mathbf{o}_{i}$ from the same and different posture classes, respectively.

Random Forest. Random forest (RF) is a classification method [4], which also provides feature importance. The basic idea is that a forest contains many decision trees, each of which is constructed by instances with randomly sampled RSSIs. The prediction is made by a majority vote of decision trees. To obtain tag importance, we split the training sets into two parts. By training the first part and predicting the second, we obtain an accuracy value. For the $j^{\text {th }}$ tag, we randomly shuffle its values in the second set and obtain another accuracy value. The difference between the two accuracy values indicate the importance of the $j^{\text {th }}$ tag.

Multinomial Logistic Regression with $\ell_{1}$ Regularization. $\ell_{1}$ regu- larization uses a penalty term that shapes the sum of the absolute values of parameters to be small, which usually leads to a sparse parameter vector. In this work, we integrate the $\ell_{1}$ regularization into linear classifier in the objective term. Given our multi-class posture recognition problem, we combine the $\ell_{1}$ regularization with multinomial logistic regression, which models the conditional probability $P_{\mathbf{w}}\left(l_{j}=\mp 1 \mid \mathbf{o}\right)$. The prime problem with $\ell_{1}$ regularization can be calculated by optimizing the log likelihood:

$$
\min _{\mathbf{w}} \sum_{k=1}^{K}\left\|\mathbf{w}_{k}\right\|_{1}-\sum_{i=1}^{n} \sum_{k=1}^{K} l_{i k} \mathbf{w}_{k}^{T} \mathbf{o}_{i}+\sum_{i=1}^{n} \log \left(\sum_{k=1}^{K} \exp \left(\mathbf{w}_{k}^{T} \mathbf{o}_{i}\right)\right)
$$

RFID tags can then be selected by considering the obtained weight vector $\mathbf{w}$.

Least Square with $\ell_{1}$ Regularization. It can be represented as:

$$
\min _{\mathbf{w} \in \mathbb{R}^{d}} \frac{1}{2}\|\mathbf{l}-\mathbf{O w}\|_{2}^{2}+\lambda\|\mathbf{w}\|_{1}
$$

where $\mathbf{l}=\left\{l_{1}, \ldots, l_{n}\right\}$ is the posture labels of training RSSI samples, $\mathbf{O}=\left\{\mathbf{o}_{1}, \ldots, \mathbf{o}_{n}\right\}$ is all training RSSI samples, $\mathbf{w}=\left[w_{1}, \ldots, w_{d}\right]^{T}$ denotes the regression coefficients, $w_{i}$ corresponds to the regression coefficient of the $i^{\text {th }}$ tag, $\lambda$ is the regularization parameter. Same as the multinomial case, $\|\mathbf{w}\|_{1}$ regularization tends to produce a sparse solution (i.e., the regression coefficients of irrelevant tags are or close to zero), which indicates the importance of each tag. We also study the $\ell_{2,1}$ regularization, which is formulated as:

$$
\min _{\mathbf{w} \in \mathbb{R}^{d}} \frac{1}{2}\|\mathbf{l}-\mathbf{O w}\|_{2}^{2}+\lambda\|\mathbf{w}\|_{2,1}
$$

where $\|\mathbf{w}\|_{2,1}=\sum_{i=1}^{n} \sqrt{\sum_{j=1}^{K} w_{i j}^{2}}$.

After performing the selection process, all tags are ordered based on their importance and a subset of tags is selected based on a userdefined threshold of top- $N(N<d)$ tags.

\subsection{Steady Posture Recognition}

We now move to the posture recognition. We use SVM (support vector machine) with linear kernel to perform steady posture classification. SVM aims at finding the decision boundary via maximizing the distance from the closet sample to the boundary hyperplane. When there are limited training data available, SVM usually outperforms the traditional parameter estimation methods which are based on the Law of Large Numbers. This is mainly due to the fact that SVM benefits from the structural risk minimization principle and the avoidance of overfitting by its soft margin. For posture recognition, SVM classifies postures based on the fact that the smaller the distance between two RSSI samples, the higher probability they belongs to a same posture. SVM method works directly with RSSI using the kernel functions. The topology implicit in sets of RSSI and the postures can be exploited in the construction of possibly non-Euclidean function spaces that are useful for posture estimation. Given the sequence of training RSSI and corresponding posture labels $\mathbf{O}=\left\{\left(\mathbf{o}_{1}, l_{i}\right), \ldots,\left(\mathbf{o}_{n}, l_{n}\right)\right\}$, where $\mathbf{o} \in \mathbb{R}^{d}$ and $l \in\{1, \ldots, K\}$, the objective function can be formulated as:

$$
\begin{array}{ll} 
& \min _{\mathbf{w}, b, \xi} \mathbf{w}^{T} \mathbf{w}+C \sum_{i=1}^{n} \xi_{i} \\
\text { s.t. } & l_{i}\left(\mathbf{w}^{T} \phi\left(\mathbf{o}_{i}\right)+b\right) \geq 1-\xi_{i}, i=1,2, \ldots, n \\
& \xi_{i} \geq 0, i=1,2, \ldots, n
\end{array}
$$

where $\xi_{i}$ is a slack variable, $C$ is the penalty of error term, $K\left(\mathbf{o}_{i}, \mathbf{o}_{j}\right)=$ $\phi\left(\mathbf{o}_{i}\right)^{T} \phi\left(\mathbf{o}_{j}\right)$ is the kernel function. 
The prime problem of optimization in Equation 7 can be converted to solve its duality using Lagrange multiplier. Thus, Equation 7 can be reformulated as:

$$
\begin{aligned}
& L(\mathbf{w}, b, \xi, \alpha, \mu)= \\
& \mathbf{w}^{T} \mathbf{w}+C \sum_{i}^{n} \xi_{i}-\sum_{i=1}^{n} \alpha_{i}\left(l_{i}\left(\mathbf{w o}_{i}+b\right)-1+\xi_{i}\right)+\sum_{i=1}^{n} \mu_{i} \xi_{i}
\end{aligned}
$$

where $\alpha=\left(\alpha_{1}, \ldots, \alpha_{n}\right)^{T}$ and $\mu=\left(\mu_{1}, \ldots, \mu_{n}\right)^{T}$ is the Lagrange multipliers. To solve Equation 8, we can maximize the minimization of duality as:

$$
\max _{\alpha, \mu} \min _{\mathbf{w}, b, \xi} L(\mathbf{w}, b, \xi, \alpha, \mu)
$$

The technical details and the mathematical theory can be found in [7]. Three widely used kernels are explored in our work, including the linear kernel, the polynomial kernel, and the Gaussian kernel:

- Linear kernel. $K\left(\mathbf{o}_{i}, \mathbf{o}_{j}\right)=\mathbf{o}_{i}^{T} \mathbf{o}_{j}$

- Gaussian kernel. $K\left(\mathbf{o}_{i}, \mathbf{o}_{j}\right)=\exp \left(-\frac{\left\|\mathbf{o}_{i}-\mathbf{o}_{j}\right\|^{2}}{2 \sigma^{2}}\right)$

- Polynomial kernel. $K\left(\mathbf{o}_{i}, \mathbf{o}_{j}\right)=\left(\mathbf{o}_{i} \cdot \mathbf{o}_{j}+1\right)^{p}$

With the model learned, we can recognize the posture class for a given testing RSSI $\mathbf{o}^{*}$.

\subsection{Posture Transition Detection}

Apart from evaluating recognition accuracy of identifying static postures using passive tag array, we specially study the performance of detecting posture transitions as RSSI values become harder to interpret due to more disturbance and noise during posture transitions (e.g., from standing straight to falling to the ground). We propose a HMM based approach, which has shown a powerful performance in handling posture sequences. Specially, our goal is to determine the conditional probability $P\left(l_{k} \mid \mathbf{o}_{i}\right)$ given a new coming sample o.

Given observation sequences of RSSI $\mathbf{O}=\left\{\mathbf{o}_{1}, \ldots, \mathbf{o}_{T}\right\}$, and posture states denoted by posture label sequence $\mathbf{l}=\left\{l_{1}, \ldots, l_{T}\right\}$, the HMM models the sequence of observable RSSI $\mathcal{O}=\left\{\mathbf{o}_{1}, \ldots, \mathbf{o}_{T}\right\}$ by assuming that there is an underlying sequence of different postures $\mathbf{l}=\left\{l_{1}, \ldots, l_{T}\right\}$ drawn from a finite posture set. In our posture transition recognition problem, each observation $\mathbf{o}_{t}$ is the RSSI vector, and each state $l_{t}$ is the posture label (e.g., sitting).

HMM makes two assumptions: i) each posture performed at $t$ only depends on its immediate previous posture at time $t-1$, and ii) each observable RSSI $\mathbf{o}_{t}$ only depends on the current performed posture $l_{t}$, which are formulated respectively as:

$$
\begin{array}{r}
p\left(l_{t} \mid l_{t-1}, \mathbf{o}_{t-1}, \ldots, l_{1}, \mathbf{o}_{1}\right)=p\left(l_{t} \mid l_{t-1}\right), t=1,2, \ldots, T \\
p\left(\mathbf{o}_{t} \mid l_{T}, \mathbf{o}_{T}, \ldots, l_{t+1}, \mathbf{o}_{t+1}, \ldots, l_{1}, \mathbf{o}_{1}\right)=p\left(\mathbf{o}_{t} \mid l_{t}\right)
\end{array}
$$

With the assumptions, we can model the joint probability of posture sequence $\mathbf{l}$ and observable RSSI sequence $\mathbf{O}$ as:

$$
p(\mathbf{l}, \mathbf{O})=\prod_{t=1}^{T} p\left(l_{t} \mid l_{t-1}\right) p\left(\mathbf{o}_{t} \mid l_{t}\right)
$$

where $p\left(l_{t} \mid l_{t-1}\right)$ is the transition probability indicating the likelihood the subject changes from posture $l_{t-1}$ to posture $l_{t}$, which is defined by considering the predefined posture transitions applications. For example, people can transit from sitting to standing, but can not transit from lying in bed to falling on ground directly,

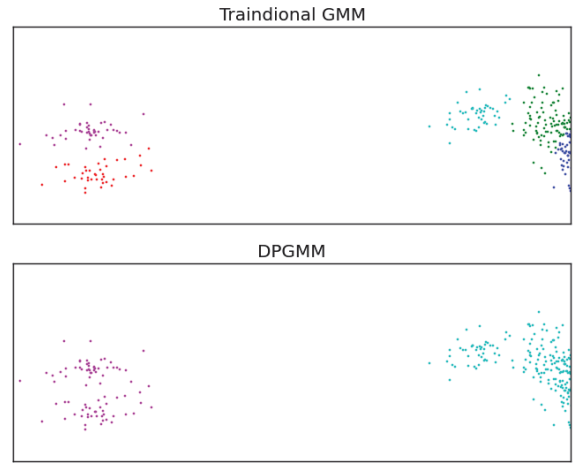

Figure 7: Comparison on traditional GMM with DPGMM over two dimensional RSSI

whilst they can transit from lying in bed to sitting then to falling on ground. We denote the state transition probability distribution as $\mathcal{A}=\left\{a_{i j}\right\}$ :

$$
a_{i j}=p\left(l_{t+1}=l_{j} \mid l_{t}=l_{i}\right)
$$

On the other hand, $p\left(\mathbf{o}_{t} \mid l_{t}\right)$ denotes the observation distribution drawn by different postures. We assume RSSI distribution generated by each posture as a Gaussian mixture model, which is a weighted sum of $m$ component Gaussian densities. It can be defined as $\mathcal{B}=\left\{b_{t}(i)\right\}$ :

$$
\begin{aligned}
b_{t}(i) & =p\left(\mathbf{o}_{t} \mid l_{t}=l_{i}\right) \\
& =\sum_{m=1}^{M_{i}} \pi_{i, m} N\left(\mathbf{o}_{t}, \mu_{i, m}, \Sigma_{i, m}\right) \\
& =p\left(\mathbf{o}_{t} \mid \mu_{m}, \Sigma_{m}\right)=\frac{1}{(2 \pi)^{D / 2} \Sigma_{m}^{1 / 2}} \exp \\
& \left(-\frac{1}{2}\left(\mathbf{o}_{t}-\mu_{m}\right)^{T} \Sigma_{m}^{-1}\left(\mathbf{o}_{t}-\mu_{m}\right)\right)
\end{aligned}
$$

where $\mathbf{o}$ is $d$ dimensional continuous RSSI observations ( $d$ is the number of tags in the deployment) and $\pi$ is the mixture weights and $p\left(\mathbf{o}_{t} \mid \mu_{m}, \Sigma_{m}\right)$ is the component Gaussian distribution.

The traditional GMM learning process with Expectation - Maximization (EM) limits to determination of how many gaussian components in the GMM. We adopt the Dirichlet Process Gaussian Mixture Model (DPGMM) in observation probability distribution in this work. It uses the Dirichlet process as a prior over the distribution of the parameters and there is no need to explicitly declare the number of components. The approximate inference algorithm uses a truncated distribution with a fixed maximum number of components, but almost always the number of components actually used depends on the data.

We use two-dimensional RSSI from our dataset to show the advantage of DPGMM over GMM (in Figure 7). GMM with EM learning splits Gaussian components arbitrarily, for example, the two clusters are eventually divided into five clusters in some convergences. Thus it does not reach a good fit even we use AIC (Akaike Information Criterion) [1] as model selection criteria, while the Dirichlet Process GMM model effectively only uses as many as needed for a good fit without defining number of guassian components, it can accurately nail down two clusters and converges to a good fit automatically in this case. More details can be referred to [3].

Our goal of detecting postures in the context of HMM is as follows. Given a sequence of RSSI observations $\mathbf{o}_{1}, \ldots, \mathbf{o}_{T}$, what is the most likely sequence of postures that produce such observations? We adopt the Viterbi algorithm to find the most likely state 


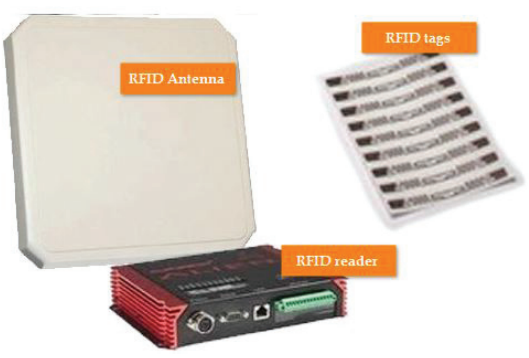

(a)
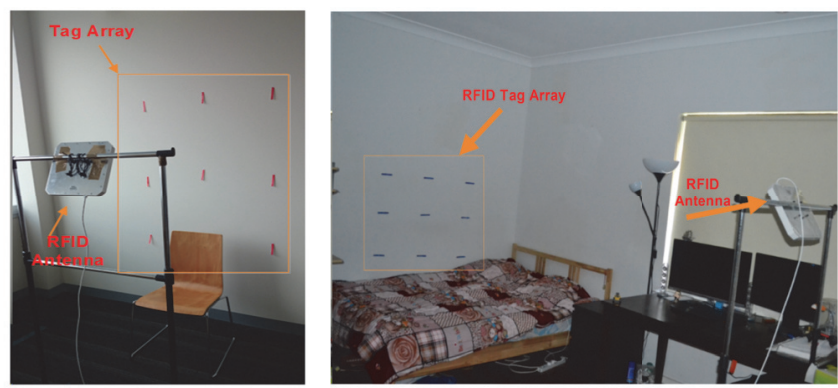

(b)

Figure 8: (a) RFID tags/reader/antenna (b) Lab setting (left) and Bedroom setting (right)

sequence in HMM. Formally, given a continuous sequence of RSSI observations $\mathbf{o}_{1} \ldots \mathbf{o}_{T}$ and learned HMM (shown in Equation 12), we aim to find the most likely posture sequence $l_{1} \ldots l_{T}$ :

$$
\delta_{t}(j)=\max _{l_{1} \ldots l_{t-1}} p\left(l_{t}=j, l_{t-1}, \ldots l_{1}, \mathbf{o}_{t}, \ldots, \mathbf{o}_{1} \mid \mathcal{A}, \mathcal{B}\right)
$$

where $\mathcal{A}$ and $\mathcal{B}$ can be calculated from Equation 13 and Equation 14. By induction, we can have:

$$
\begin{aligned}
& \delta_{1}(j)=b_{1}\left(\mathbf{o}_{j}\right) \\
& \delta_{t+1}(j)=\max _{1 \leq j \leq N} \delta_{t-1}(j) a_{i j} b_{t}\left(\mathbf{o}_{t+1}\right), j=1, \ldots, K
\end{aligned}
$$

\section{EXPERIMENTS}

This section reports our experimental studies in both lab and realworld residential environments. First, we describe the experimental settings, and then we report the results.

\subsection{Experimental Settings}

Hardware Setup. We used one Alien 9900+ RFID reader, one circular antenna and Squig inlay passive RFID tags in our experiments (see Figure 8 (a)). The tag array containing nine tags was placed at a $3 \times 3$ grid points on a wall where each grid is roughly $0.58 \mathrm{~m} \times 0.58 \mathrm{~m}$. We name this wall the active testing area in this paper. The antenna was arranged in $\approx 1.3 \mathrm{~m}$ height facing the active testing area in $\approx 70^{\circ}$ (see Figure $8(\mathrm{~b})$ ). The subject performed different predefined postures between the wall and the antenna, and the corresponding sequence of RSSI were collected at a sampling rate of 0.5 second.

Sampling Rate. Passive RFID tags tend to be noisy even in a lab environment. For example, one challenge in existing RFID systems is false negative readings, caused by missed detections (i.e., a tag in the antenna's reading range not detected). In addition, RSSI data is much sensitive to environments, e.g., some disturbance from environment can cause RSSI fluctuations. Appropriate sampling rates can reduce the aforementioned problems. Too small sampling rates make our method more sensitive to the noise of RFID readings, while too big sampling rates blur the inter-class posture boundaries. In our implementation, we collected the continuous RSSI data streams at the sampling rate of $\approx 0.5$ second.

Data Acquisition. We ran a pilot study to evaluate the performance of our system. For collecting the training dataset, we conducted a series of experiments in which a subject entered the active testing area and performed various pre-arranged postures, such as standing, lying on ground, lying in bed, and falling. Three subjects (two males and one female) participated in the experiments and each performed the set of 12 fine-grained postures (Figure 9). The subjects

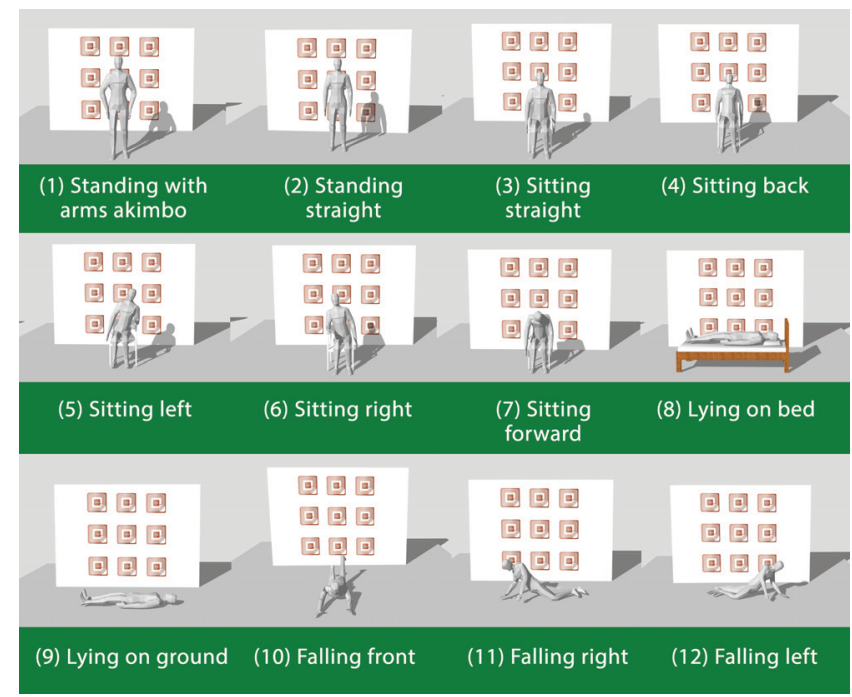

Figure 9: Predefined orientation-sensitive postures

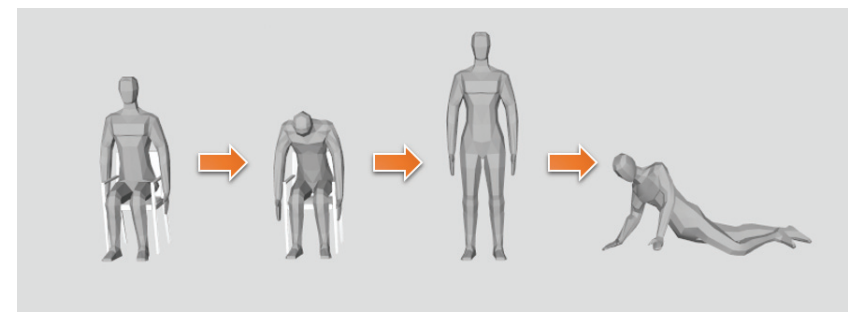

Figure 10: An example of posture transition sequence

also performed different predefined posture sequences for evaluating posture transition detection. The timing of all the movements was determined beforehand so that the data can later be compared to the actual timing of the postures.

The task of the steady posture classification is to model how the signal strengths are distributed when the subject performs different postures. Each subject stands in the active testing area which is between the antenna and the wall deployed with passive RFID tags. We first measured the RSSI values for all tags when the testing area is empty. Then each subject stood in the area and performed the 12 predefined postures.

For collecting the posture transition dataset, we designed eight different posture sequences to simulate the posture sequences in real world (see Figure 10) and collected them using two strategies. In the first strategy, the subject performed and held each posture for 


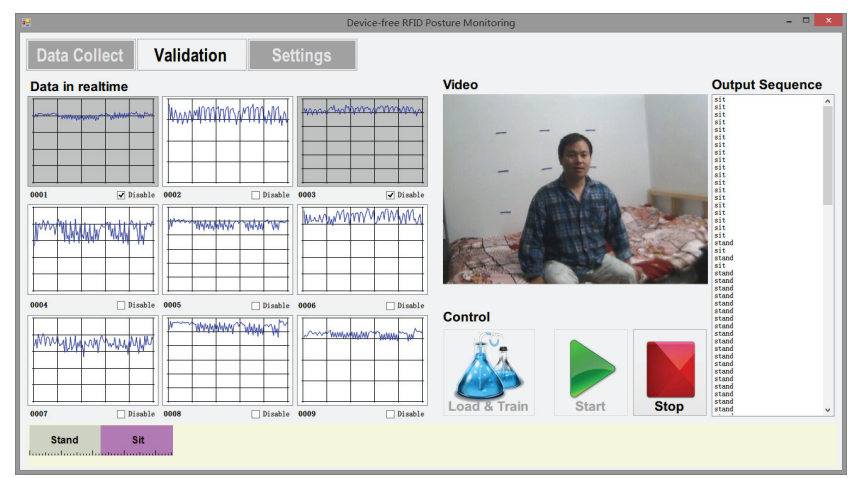

Figure 11: System interface

30 seconds and then performed next posture in the order as predefined in the sequence. In the second strategy, the subject performed and held each posture for 60 seconds and then performed the next posture in the order as predefined in the sequence. Figure 11 shows a system interface for our proposed approach, which integrates data collection, annotation and monitoring.

\subsection{Results}

To evaluate the effectiveness of the proposed tag selection, we adopted a person-dependent 10 -fold cross-validation strategy. For the person-dependent evaluation, we use partial samples of a subject for testing and use the remaining samples of the same participant for training.

\subsubsection{Impact on Tag Selection}

To evaluate the impact of tag selection, we sorted the tag importance calculated from six selection approaches (Section 3.1) in descend order, and compared the recognition accuracy using SVM with linear kernel by choosing top $N$ tags ( $N$ is from 1 to 9 (full set)). Figure 12 (a) shows the comparison results over top $N$ tags in the lab environment, and Figure 13 (b) shows the results in the bedroom environment.

In both cases, the performance is influenced by the selected tags. Posture classification accuracy does not improve much after top 5 selected tags using all selection criteria, and reaches the best point (99.18\%) with Relief-F selection when top-7 tags are selected compared to $99.04 \%$ without tag selection (full set of tags). In the bedroom case, the impact of tag selection on performance is more obvious. The accuracy is the best when only seven tags are selected, and the performance even slightly drops when more tags are added. From the results, we can see that the tag selection does improve the overall performance in both lab and bedroom environments by distinguishing the salient tags, only subset of intuitively placed tags shows their usefulness and discrimination via implicating the intraperson variability on different postures. The rest of tags degrade the overall performance due to failing to capture the inter-class and intra-class variability. Figure 13 shows an example of optimal tag deployments from our experiments.

\subsubsection{Steady Posture Classification}

To study the feasibility of our approach and its sensitivity to the size of training data after selecting tags, we further evaluated the posture classification with varying training ratios in terms of tag selection and no tag selection. As shown in Figure 14, our approach performs well even only with $10 \%$ the training size, the accuracy reaches over $90 \%$ in both cases. The accuracy increases with larger training size. However, when the training size is around

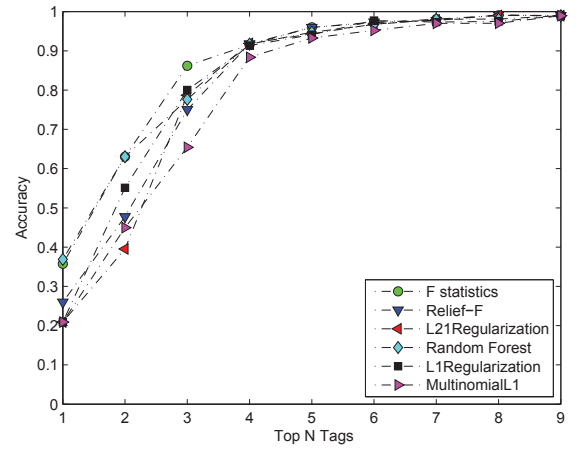

(a) Lab environment

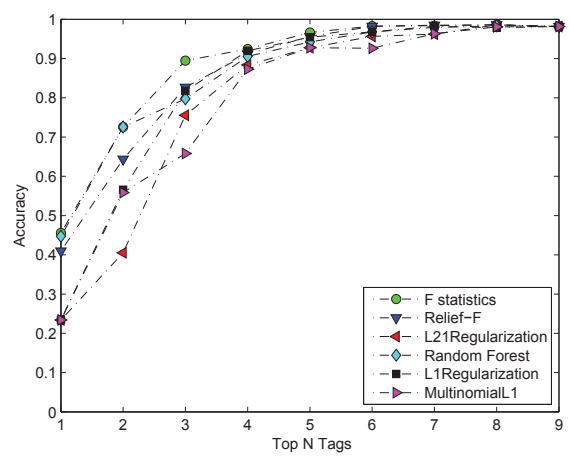

(b) Bedroom environment

Figure 12: Posture classification comparison with Top $N$ tag selection in (a) lab and (b) bedroom environments

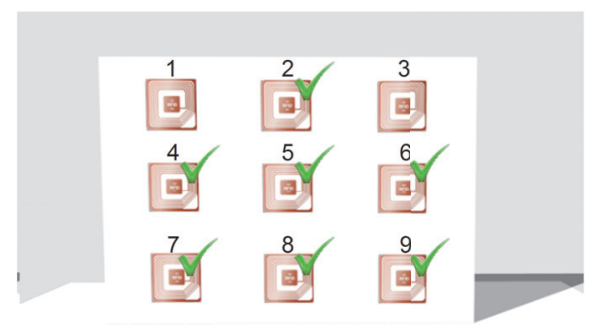

Figure 13: An example on tags selection

$60 \% \sim 70 \%$, the accuracy begin to decrease. We can see that the overall accuracy is consistently better with the tag selection strategy compared with the case without tag selection.

We look closely at the results of the confusion matrices in both lab and bedroom cases with the selected subset of tags and $10 \%$ training ratio given in Table 1 and Table 2, respectively. Generally, only a few samples of postures, i.e., standing free (with ID of 1) and standing straight (with ID of 2), misclassified in the lab environment. In the bedroom environment, falling right (with ID of 11) and falling left (with ID of 12) are misclassified, whilst they are accurately classified in the lab environment. It should be noted that the performance on classifying orientation-sensitive postures still reaches over $98 \%$ in the bedroom environment.

\subsubsection{Posture Transition Detection}

To evaluate the performance of detecting sequential postures, we 


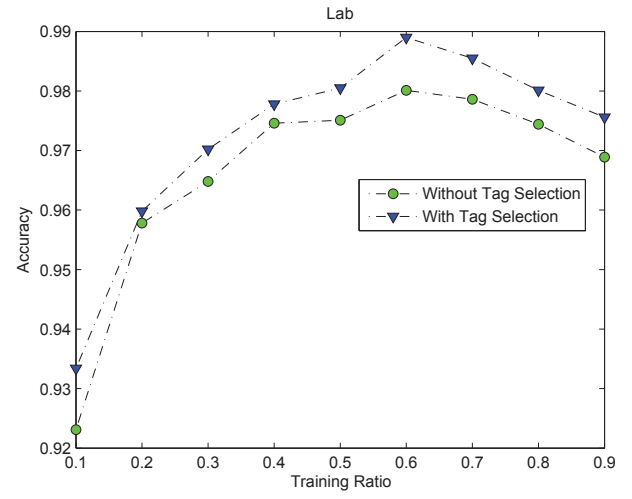

(a) Lab environment

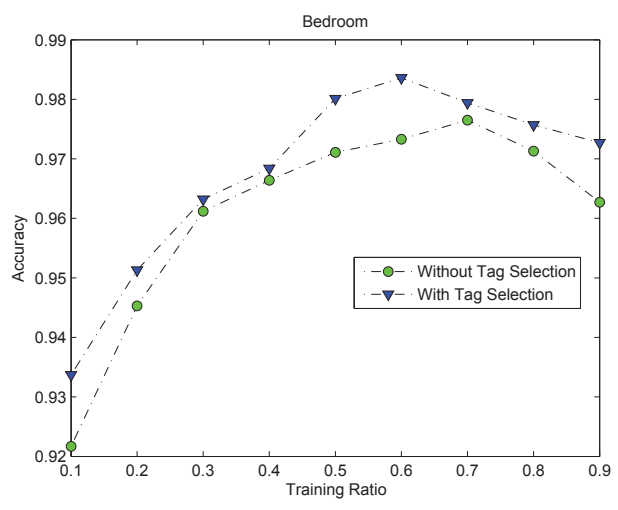

(b) Bedroom environment

Figure 14: Accuracy comparison with and without tag selection using different training sizes: (a) lab and (b) bedroom

performed posture classification over a series of posture transitions and measured how accurately our approach can recognize a posture given new coming RSSI values, as well as how timely our approach can recognize the posture.

Passive RFID tags are highly sensitive to disturbance, especially when posture changes. The RSSI fluctuation result from posture transition exhibits some uncertainty. To cope with the impact of this disturbance, we adopted a forward calibration mechanism to calibrate the RSSI streams before detecting posture change [22]. We used a sliding time averaging window to smooth RSSI values. The calibrated RSSI stream $\mathbf{o}_{t}^{\prime}$ at time $t$ can be calculated as:

$$
\hat{\mathbf{o}}_{t}{ }_{t}=\frac{\sum_{i=t}^{t+|w|-1} \mathbf{o}_{i}^{\prime}}{|w|}
$$

where $|w|$ is the window size. To determine the best window size in this work, we evaluated the performance of both lab and bedroom settings with and without tag selection strategy by varying the window size. Figure 15 shows the results.

From the figure, we can see that the performance does not consistently improve when increasing window size, instead, when the window size is 2 , the performance in both settings reached the best result. We further compared the performance in terms of different duration a posture is held. Figure 15 shows the results under two durations (30 and 60 seconds) with and without tag selection. From the results, we can see that the longer the posture is held by the subject, the better accuracy can be achieved. The reason is that a longer posture holding time can eliminate both inter-class and intra-class variations, to which RSSI are especially sensitive in rec-
Table 1: Confusion matrix with tag selection in lab

\begin{tabular}{|c|c|c|c|c|c|c|c|c|c|c|c|c|}
\hline ID & 1 & 2 & 3 & 4 & 5 & 6 & 7 & 8 & 9 & 10 & 11 & 12 \\
\hline \hline 1 & 35 & 2 & & & & & & & & & & \\
\hline 2 & 1 & 34 & & & & & & & & & & \\
\hline 3 & & & 39 & & & & & & & & & \\
\hline 4 & & & & 36 & & & & & & & & \\
\hline 5 & & & & & 36 & & & & & & & \\
\hline 6 & & & & & & 36 & & & & & & \\
\hline 7 & & & & & & & 39 & & & & & \\
\hline 8 & & & & & & & & 36 & & & & \\
\hline 9 & & & & & & & & & 37 & & & \\
\hline 10 & & & & & & & & & & 36 & & \\
\hline 11 & & & & & & & & & & & 36 & \\
\hline 12 & & & & & & & & & & & & 36 \\
\hline
\end{tabular}

Table 2: Confusion matrix with tag selection in bedroom

\begin{tabular}{|c|c|c|c|c|c|c|c|c|c|c|c|c|}
\hline ID & 1 & 2 & 3 & 4 & 5 & 6 & 7 & 8 & 9 & 10 & 11 & 12 \\
\hline \hline 1 & 35 & 1 & & & & & & & & & & \\
\hline 2 & 1 & 34 & & & & & & & & & & \\
\hline 3 & & & 36 & & & & & & & & & \\
\hline 4 & & & & 36 & & & & & & & & \\
\hline 5 & & & & & 36 & & & & & & & \\
\hline 6 & & & & & & 36 & & & & & & \\
\hline 7 & & & & & & & 39 & & & & & \\
\hline 8 & & & & & & & & 36 & & & & \\
\hline 9 & & & & & & & & & 37 & & & \\
\hline 10 & & & & & & & & & & 36 & & \\
\hline 11 & & & & & & & & & & & 35 & 2 \\
\hline 12 & & & & & & & & & & & 1 & 35 \\
\hline
\end{tabular}

ognizing postures. We also can see that the performance using the tag selection strategy significantly outperforms the one without tag selection. The results from steady posture classification and posture transitions detection consistently indicate that an optimal subset of tags can more discriminatingly recognize postures compared with full set of tags. The subset tags have the dominant impact.

Fast detecting posture transition is critical, particularly for aged care applications. For example, for fall detection, we can send an alert and notify caregivers as quickly as possible to offer medical assistance for elderly people when a fall occurs. The results from our experiments show that the proposed method promptly detects posture transition. Interested readers are referred to our demo ${ }^{1}$.

\subsubsection{Recognition Delay}

We now evaluate how quickly our system detects the transition of a person from one posture to the other. Our system has 3.5 seconds recognition latency, which results from two main factors. Firstly, our system evaluates subject's postures every 0.5 seconds using the latest 2 seconds of RSSI stream. In other words, if the current system time is at timestamp $t$, our system will produce the predicted posture in the $[t-2, t-1]$ seconds, and $[t-1, t]$ seconds is used to backtrack check if the predicted label complies with predefined rules. For instance, assume that the label is estimated as: lying on bed at $[t-2, t-1]$ interval, if the predicted label in interval $[t-1, t]$ is nobody, our system will determine the subject is still lying on bed. Secondly, the RSSI collector is programmed with a timer to poll the RSSI with a predefined order of transmission, which needs to take around 1 second to complete a new measurement with no workarounds. From Figure 16, we can see that our system promptly detects posture transition with slight latency.

We can see that the overall performance at home environment is a little bit lower than the lab environment (due to furnitures etc). However, it still achieves over $98 \%$ accuracy for steady posture classification and $70 \%$ for the overall posture transition detection.

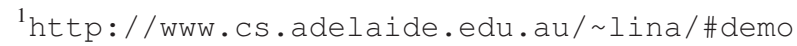




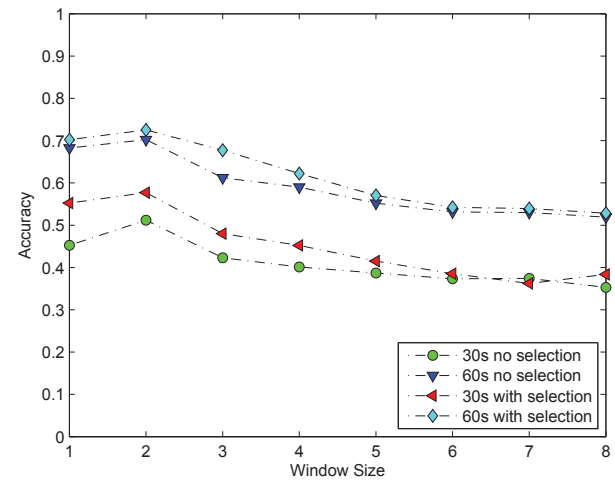

(a)

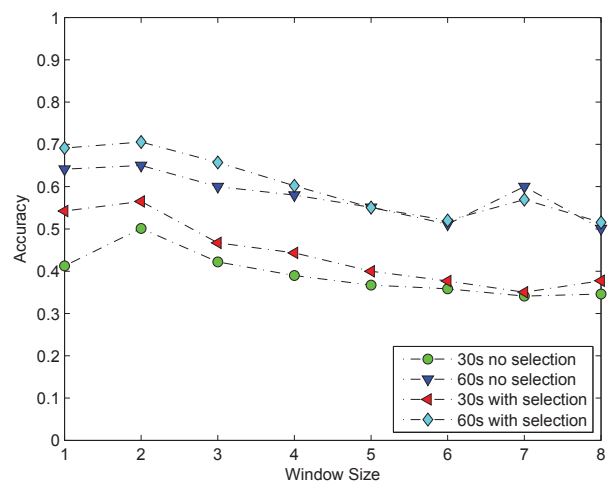

(b)

Figure 15: Performance comparison on different window sizes using 30s and 60s strategies without and with tag selection in (a) lab environment, (b) bedroom environment

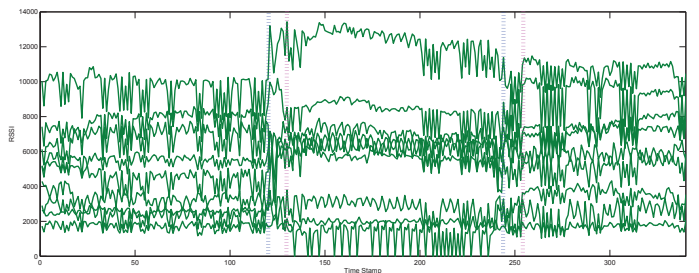

Figure 16: Recognition latency: blue dot vertical line indicates the ground-truth time point of posture transition, pink dot vertical line indicates the recognition time point detected by our system.

\section{RELATED WORK}

The goal of activity recognition is to detect human physical activities from the data collected through various sensors. There are generally two main research directions: i) to augment people, on whom sensors and RFID tags are attached, and ii) to augment the environment, where sensors are deployed inside the environment and people do not have to carry them.

Wearable sensors such as accelerometers and gyroscopes are commonly used for recognizing activities [15, 2, 18]. For example, the authors in [12] design a network of three-axis accelerometers distributed over a user's body. Activities can then be inferred by learning information provided by accelerometers about the orien- tation and movement of the corresponding body parts. However, such approaches have obvious disadvantages including discomfort of wires attached to the body as well as the irritability that comes from wearing sensors for a long duration. Recently, researchers are exploring smart phones equipped with accelerometers and gyroscopes to recognize activities and gesture patterns $[16,17]$. $\mathrm{Kr}$ ishnan et al. [14] propose an activity inference approach based on motion sensors installed in a home environment.

Apart from sensors, RFID has been increasingly explored in the area of human activity recognition. Some research efforts propose to realize activity recognition by combining RFID passive tags with traditional sensors (e.g., accelerometers). In this way, daily activities are inferred from the traces of object usage via various classification algorithms such as Hidden Markov Model, boosting and Bayesian networks [24, 5]. Other efforts dedicate to exploit the potential of using "pure" RFID techniques for activity recognition. For example, Wang et al. [26] use RFID radio patterns to extract both spatial and temporal features, which are in turn used to characterize various activities. However, these research efforts require people to carry RFID tags or even readers (e.g., wearing a bracelet).

There are recent research efforts focusing on exploring devicefree activity recognition. Such approaches exploit radio transmitters installed in environments, and people are free from carrying any receiver or transmitter. Most device-free approaches concentrate on analyzing and learning distribution of received signal strength or radio links. Youssef et al. [28] propose to localize people by analyzing wireless signal strength moving average and variance. Zhang et al. [29] develop a tag-free sensing approach using RFID tag array. However, most of these efforts focus on localization and tracking, not on activity recognition. Only very recently, the authors of [10] and [23] propose device-free activity recognition using sensor arrays. Compared to these work, our passive RFID-based system has many advantages such as low cost and maintenance free. To the best of our knowledge, our work is the very first of few on investigating device-free human activity recognition using passive RFID tags.

\section{CONCLUSION}

In this paper, we proposed a device-free posture monitoring system for elderly people, by exploiting low-cost passive RFID tags. We focus our study on tag configuration issues, especially tag placement and selection, for achieving the best trade-off between performance and cost. We systematically study these issues by using different configuration settings and applying various tag selection methods. We also propose a Dirichlet Process Gaussian Mixture Model with the Hidden Markov model to recognize different postures. We conducted extensive empirical studies and our findings are briefly summarized as follows.

- Our system detects 12 orientation-sensitive postures (see Figure 9), with an accuracy of $99 \%$ and $72 \%$ in terms of steady posture recognition and posture transition detection, respectively, in a lab environment, and over $98 \%$ and $70 \%$, in a real-life home environment.

- It is interesting to find out that more tags deployed in the testing area do not help improve the performance, which actually decreases the recognition accuracy. From our knowledge, our work is the first to investigate a number of possible techniques for tag configuration in posture recognition.

There are two main directions for our future work. Firstly, we will further study tags correlation in terms of temporal and spatial features, e.g., strength variation and coverage, etc, which can be 
used to build a more robust tag coverage model. Secondly, we view our work presented in this paper the first step toward recognizing high-level activities. There are in general three types of activities on top of postures: i) action, which consists of multiple postures for a single person with temporal dimension, e.g., "walking"; ii) interaction, which involves two or more persons, e.g., two people are shaking hands; and iii) group activity, which is performed by a group of people, e.g., having a meeting. Identifying these complex activities is another main goal of our future work.

\section{REFERENCES}

[1] H. Akaike. A new look at the statistical model identification. Automatic Control, IEEE Transactions on, 19(6):716-723, 1974.

[2] L. Bao and S. S. Intille. Activity recognition from user-annotated acceleration data. In Proceedings of 2 nd International Conference on Pervasive Computing (PERVASIVE), pages 1-17. Springer, 2004.

[3] D. M. Blei, M. I. Jordan, et al. Variational inference for dirichlet process mixtures. Bayesian analysis, 1(1):121-143, 2006.

[4] L. Breiman. Random forests. Machine Learning, 45(1):5-32, 2001.

[5] M. Buettner, R. Prasad, M. Philipose, and D. Wetherall. Recognizing daily activities with rfid-based sensors. In Proc. of 11th ACM Intl. Conference on Ubiquitous Computing (UbiComp), pages 51-60, 2009.

[6] D. Cook and M. Schmitter-Edgecombe. Assessing the quality of activities in a smart environment. Methods of Information in Medicine, 48(5):480, 2009.

[7] C. Cortes and V. Vapnik. Support-vector networks. Machine Learning, 20(3):273-297, 1995.

[8] J. Han, C. Qian, D. Ma, X. Wang, J. Zhao, P. Zhang, W. Xi, and Z. Jiang. Twins: device-free object tracking using passive tags. In Proc. of IEEE Intl. Conference on Computer Communications (INFOCOM), 2014.

[9] T. He et al. Vigilnet: An integrated sensor network system for energy-efficient surveillance. ACM Transactions on Sensor Networks (TOSN), 2(1):1-38, 2006.

[10] J. Hong and T. Ohtsuki. Ambient intelligence sensing using array sensor: device-free radio based approach. In Proc. of ACM Conference on Pervasive and Ubiquitous Computing Adjunct Publication, 2013.

[11] S. Intille et al. Using a live-in laboratory for ubiquitous computing research. In Proc. of Intl. Conf. on Pervasive Computing (PERVASIVE). 2006.

[12] N. Kern, B. Schiele, H. Junker, P. Lukowicz, and G. Tröster. Wearable sensing to annotate meeting recordings. Personal and Ubiquitous Computing, 7(5):263-274, 2003.

[13] N. Kern, B. Schiele, and A. Schmidt. Multi-sensor activity context detection for wearable computing. In Proc. of the 1st European Symposium on Ambient Intelligence (EUSAI), pages 220-232. 2003.

[14] N. C. Krishnan and D. J. Cook. Activity recognition on streaming sensor data. Pervasive and Mobile Computing, 10:138-154, 2014.

[15] N. C. Krishnan and S. Panchanathan. Analysis of low resolution accelerometer data for continuous human activity recognition. In Proceedings of IEEE International Conference on Acoustics, Speech and Signal Processing (ICASSP), pages 3337-3340. IEEE, 2008.
[16] J. R. Kwapisz, G. M. Weiss, and S. A. Moore. Activity recognition using cell phone accelerometers. ACM SIGKDD Explorations Newsletter, 12(2):74-82, 2011.

[17] N. D. Lane et al. Bewell: A smartphone application to monitor, model and promote wellbeing. In Proc. of 5th Intl. ICST Conference on Pervasive Computing Technologies for Healthcare, pages 23-26, 2011.

[18] P. Lukowicz, H. Junker, M. Stäger, T. von Bueren, and G. Tröster. Wearnet: A distributed multi-sensor system for context aware wearables. In Proceedings of ACM International Conference on Pervasive and Ubiquitous Computing (UbiComp), pages 361-370. Springer, 2002.

[19] D. Minnen, T. Starner, I. Essa, and C. Isbell. Discovering characteristic actions from on-body sensor data. In Proc. of 10th IEEE International Symposium on Wearable Computers (ISWC), pages 11-18, 2006.

[20] M. Philipose et al. Inferring activities from interactions with objects. IEEE Pervasive Computing, 3(4):50-57, 2004.

[21] M. Seifeldin, A. Saeed, A. E. Kosba, A. El-Keyi, and M. Youssef. Nuzzer: A large-scale device-free passive localization system for wireless environments. IEEE Transactions on Mobile Computing (TMC), 12(7):1321-1334, 2013.

[22] M. Seifeldin and M. Youssef. A deterministic large-scale device-free passive localization system for wireless environments. In Proc. of the 3rd Intl. Conference on Pervasive Technologies Related to Assistive Environments, 2010.

[23] S. Sigg, M. Scholz, S. Shi, Y. Ji, and M. Beigl. Rf-sensing of activities from non-cooperative subjects in device-free recognition systems using ambient and local signals. IEEE Transactions on Mobile Computing (TMC), 13(4):907-920, 2014.

[24] M. Stikic et al. Adl recognition based on the combination of rfid and accelerometer sensing. In Proc. of Intl. Conference Pervasive Computing Technologies for Healthcare, 2008.

[25] S. Wagner et al. On optimal tag placement for indoor localization. In Proc. of IEEE Intl. Conference on Pervasive Computing and Communications (PerCom), pages 162-170, 2012.

[26] L. Wang, T. Gu, H. Xie, X. Tao, J. Lu, and Y. Huang. A wearable rfid system for real-time activity recognition using radio patterns. In Proc. of the 10th International Conference on Mobile and Ubiquitous Systems: Computing, Networking and Services (MobiQuitous), 2013.

[27] J. A. Ward et al. Activity recognition of assembly tasks using body-worn microphones and accelerometers. IEEE Trans. on Pattern Analysis and Machine Intelligence (TPAMI), 28(10):1553-1567, 2006.

[28] M. Youssef, M. Mah, and A. Agrawala. Challenges: device-free passive localization for wireless environments. In Proc. of 13th ACM Intl. Conference on Mobile Computing and Networking (MobiCom), 2007.

[29] D. Zhang, J. Zhou, M. Guo, J. Cao, and T. Li. Tasa: tag-free activity sensing using rfid tag arrays. IEEE Transactions on Parallel and Distributed Systems (TPDS), 22(4):558-570, 2011.

[30] D. Zhang et al. Rass: A real-time, accurate and scalable system for tracking transceiver-free objects. In Proc. of IEEE Intl. Conference on Pervasive Computing and Communications (PerCom), 2011. 